\title{
Isotope energy shift of luminescence in hydrogen- and deuterium-terminated porous silicon
}

\author{
T MATSUMOTO*, S V NAIR and Y MASUMOTO ${ }^{\dagger}$ \\ Single Quantum Dot Project, ERATO, Japan Science and Technology Corporation, 5-9-9 Tokodai, \\ Tsukuba 300-2635, Japan \\ ${ }^{\dagger}$ Also at Institute of Physics, University of Tsukuba, Tsukuba 305-8571, Japan
}

\begin{abstract}
We review our recent results on the comparative studies between hydrogen-(H-PS) and deuterium(D-PS) terminated porous Si nanostructures. The photoluminescence (PL) spectrum of D-PS was different from that of H-PS despite both H-PS and D-PS showing the same structure and the same absorption spectrum. We quantitatively discuss these results based on the trapping of conduction electrons at a surface Si-H or Si-D bond supported by a tight binding calculation. In addition to these basic results, we also show that the replacement of hydrogen with deuterium reduces the degradation not only of PL but also of electroluminescence. We also discuss the luminescence degradation mechanism from the point of view of a photoinduced oxidation model.
\end{abstract}

Keywords. Isotope energy shift; hydrogen; deuterium; porous silicon.

\section{Introduction}

Nanostructural silicon formed by electrochemical anodization has attracted much interest recently because it exhibits strong visible photoluminescence (PL) at room temperature (Canham 1990). Although a great deal of effort has been made to elucidate the origin of photoluminescence, much of the uncertainty still remains due to the difficulty of physical characterization of the $\mathrm{Si}$ nanostructures.

Isotope substitution is a powerful tool to study molecular terminations, kinetics of atoms or molecules, or the interaction between nuclei and extra nuclear electrons. Especially, hydrogen and its isotope are widely used to study the surface structure (Chabal and Raghavachari 1984) and the diffusion of hydrogen in crystalline Si (Pankov and Johnson 1991). Because a huge amount of terminating hydrogen is supplied from hydrofluoric acid (HF) in electrochemical anodization process (Gupta et al 1988; Hardeman et al 1985), the surface terminations of Si nanostructure can be changed from hydrogen to deuterium by using an isotope electrolyte such as deuterofluoric (DF) acid.

In this paper, we review our recent results on the comparative studies between hydrogen-(H-PS) and deuterium-(D-PS) terminated porous Si nanostructures (Matsumoto et al 1997a, b). Firstly, we will describe the results of structural characterization using scanning electron microscope (SEM) analysis, transmission electron microscope (TEM) analysis, Raman spectroscopy and Fourier transform infrared spectroscopy. Secondly, we

*Author for correspondence will show optical properties such as absorption and photoluminescence (PL) spectrum. Thirdly, we will analyze these experimental results based on a tight-binding calculation.

In addition to the above fundamental results, we will introduce the result that the replacement of hydrogen with deuterium greatly reduces the PL degradation of PS. Thus, this material provides not only information regarding the physical understanding of the luminescent mechanism but also a method of stabilization of electroluminescence (EL) devices. Lastly, we will compare the electronic properties of light emitting diodes (LED) based on D-PS with those of diodes based on H-PS.

\section{Experimental}

\subsection{Electrochemical anodization}

H-PS and D-PS were formed by electrochemical anodization. All of the PS samples were formed on $3-5 \Omega \mathrm{cm}$ $p$-type (100) oriented $\mathrm{Si}$ wafers. Thin $\mathrm{Al}$ films were evaporated on the back of the wafers to form a good ohmic contact and a platinum mesh was used for the anodization cathode. The H-PS was fabricated in the dark to avoid oxidation using HF-ethanol solution ( $\mathrm{HF}: \mathrm{H}_{2} \mathrm{O}: \mathrm{C}_{2} \mathrm{H}_{5} \mathrm{OH}=1: 1: 2$ ) by applying a positive bias to the substrate with a current density in the range $20 \mathrm{~mA} / \mathrm{cm}^{2}$ for $5 \mathrm{~min}$. The D-PS were also fabricated with the same condition except for the use of DF-ethanol D6 solution (DF : $\mathrm{D}_{2} \mathrm{O}: \mathrm{C}_{2} \mathrm{D}_{5} \mathrm{OD}=1: 1: 2$ ). Both porous layers (H-PS and D-PS) have the same $(1 \mu \mathrm{m} / \mathrm{min})$ thickness. 


\subsection{SEM observations}

Figures 1a and b are SEM micrographs which show the surface images of H-PS and D-PS with current densities of $20 \mathrm{~mA} / \mathrm{cm}^{2}$. These SEM observations were performed using Hitachi S-5000-H system operated at $10 \mathrm{keV}$. A large number of $\mathrm{Si}$ nanocrystals, $<10 \mathrm{~nm}$, which is smaller than the resolution of this system, can be observed for both H-PS and D-PS. Both layers show almost the same Si-crystal structure from $\mu \mathrm{m}$ - to $10 \mathrm{~nm}$ region.

\subsection{TEM observations}

Figures $2 \mathrm{a}$ and $\mathrm{b}$ show the size distributions of nanometer crystallites in H-PS and D-PS observed by TEM micrographs. TEM observations were performed using JEOL 2010 system operated at $200 \mathrm{keV}$. Our TEM
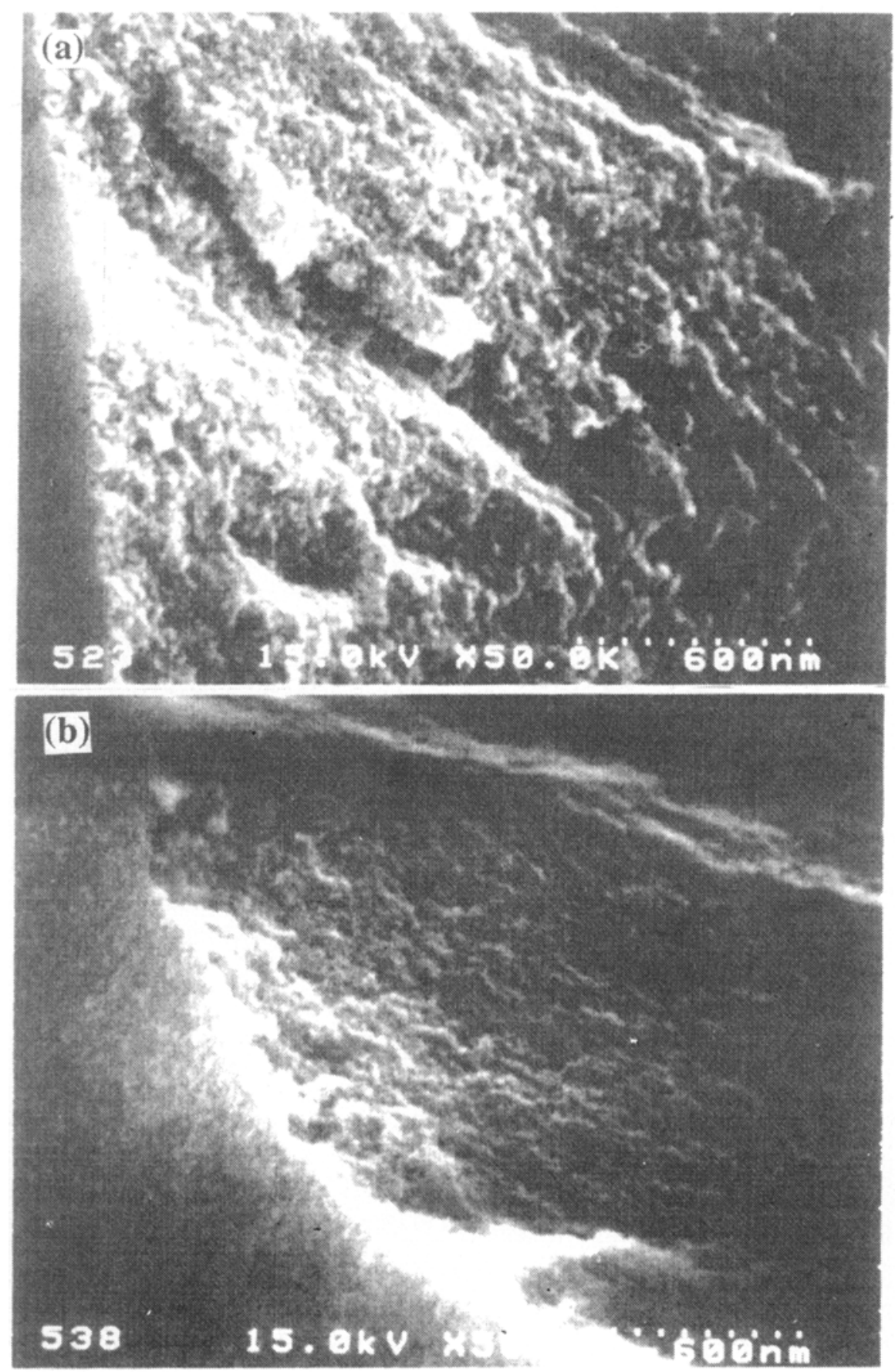

Figure 1. SEM micrographs of the surface images of (a) H-PS and (b) D-PS. 
observations show that the statistically averaged diameters of H-PS and D-PS were $3.1 \mathrm{~nm}$ and $3.3 \mathrm{~nm}$, as shown in figure 2. The lattice images of nanocrystallites observed by our TEM reveal the atomic spacing to be about $0.3 \mathrm{~nm}$, which corresponds to the silicon (111) interplanar spacing. The TEM observations confirm that the Sicrystallite-structure of D-PS is almost the same as that of H-PS at the nanometer sized region.
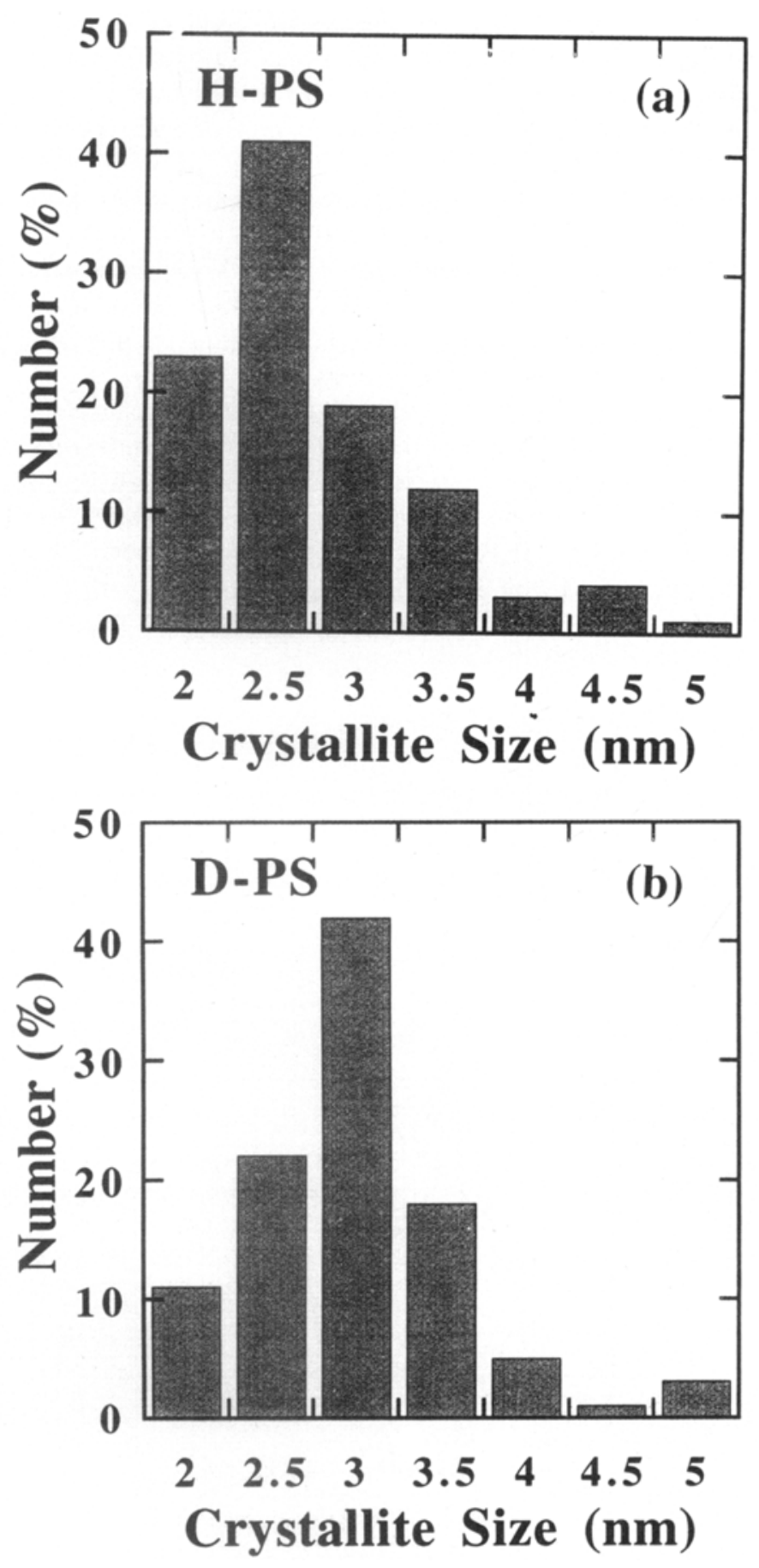

Figure 2. Size distribution (TEM) of nanometer crystallites of (a) H-PS and (b) D-PS.

\subsection{Raman analysis}

Figures $3 \mathrm{a}$ and $\mathrm{b}$ show Raman spectra of H-PS and D-PS of as-prepared samples. Broad and down shifted
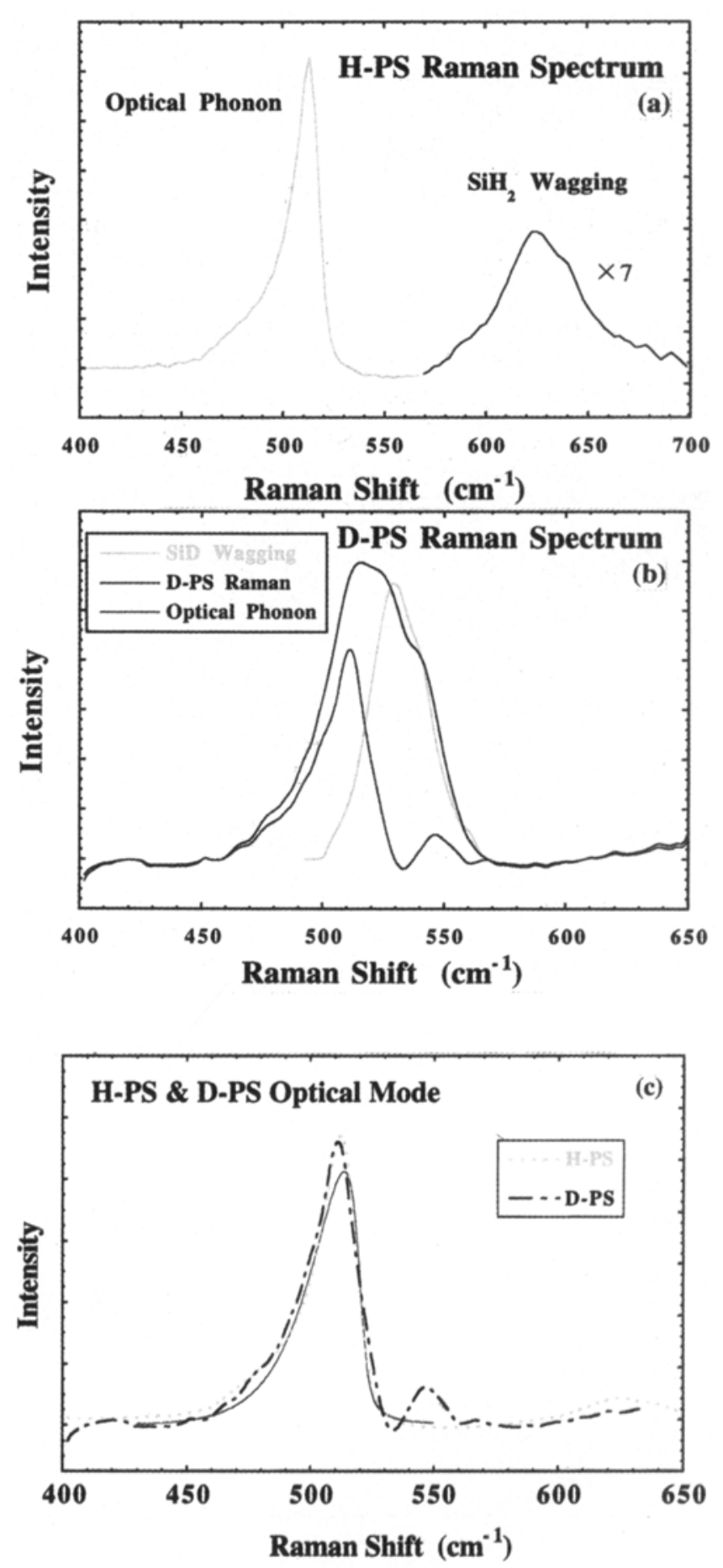

Figure 3. Raman spectrum of (a) H-PS and (b) D-PS. The red line is the $\mathrm{SiD}_{2}$-wagging mode and the green line is the extracted Si-LO Raman spectrum. (c) Comparative LO phonon spectrum between H-PS (dotted red line) and D-PS (broken blue line). The solid line shows the theoretically fitted curves using the spatial correlation model. 
spectrum was observed in H-PS compared with those in bulk Si crystals-the spectrum peaks near $515 \mathrm{~cm}^{-1}$ with a width (full width at half maximum) of $17 \mathrm{~cm}^{-1}$ for H-PS. In addition to this $\mathrm{LO}$ phonon peak, $\mathrm{SiH}_{2}$ wagging mode was also observed at around $625 \mathrm{~cm}^{-1}$. However, the observed Raman spectrum of D-PS shows a different shape from that of H-PS due to the isotope shift of the wagging mode as shown in figure $3 \mathrm{~b}$-the wagging mode of $\mathrm{Si}-\mathrm{D}_{2}$ shows an isotope shift from $625 \mathrm{~cm}^{-1}$ to $530 \mathrm{~cm}^{-1}$, which overlaps the LO Raman spectrum of the nanocrystal. Therefore, it is necessary to separate the total D-PS spectrum into the Si-LO Raman band and the $\mathrm{Si}-\mathrm{D}_{2}$-wagging mode. Figure $2 \mathrm{~b}$ shows the measured Raman spectrum of the D-PS (blue line), the overlapped $\mathrm{Si}-\mathrm{D}_{2}$-wagging mode (red line), and the extracted Si-LO Raman band (green line). The extracted Si-LO Raman spectrum of D-PS has almost the same shape as that of H-PS as shown in figure $2 c$, which indicates that the size of the nanostructure of D-PS is almost the same as that of H-PS. The average size of $\mathrm{Si}$ nanostructure can be estimated by using the spatial correlation model (Richter et al 1981; Campbell and Fauchet 1984). Both the LO Raman spectra can be characterized by an average diameter of $3.5 \mathrm{~nm} \mathrm{Si}$ particles. The results obtained here show that the nanostructure of D-PS is almost the same as that of H-PS, which agrees well with the TEM observations.

\subsection{Optical properties}

Figure 4 shows the optical absorption and the photo-
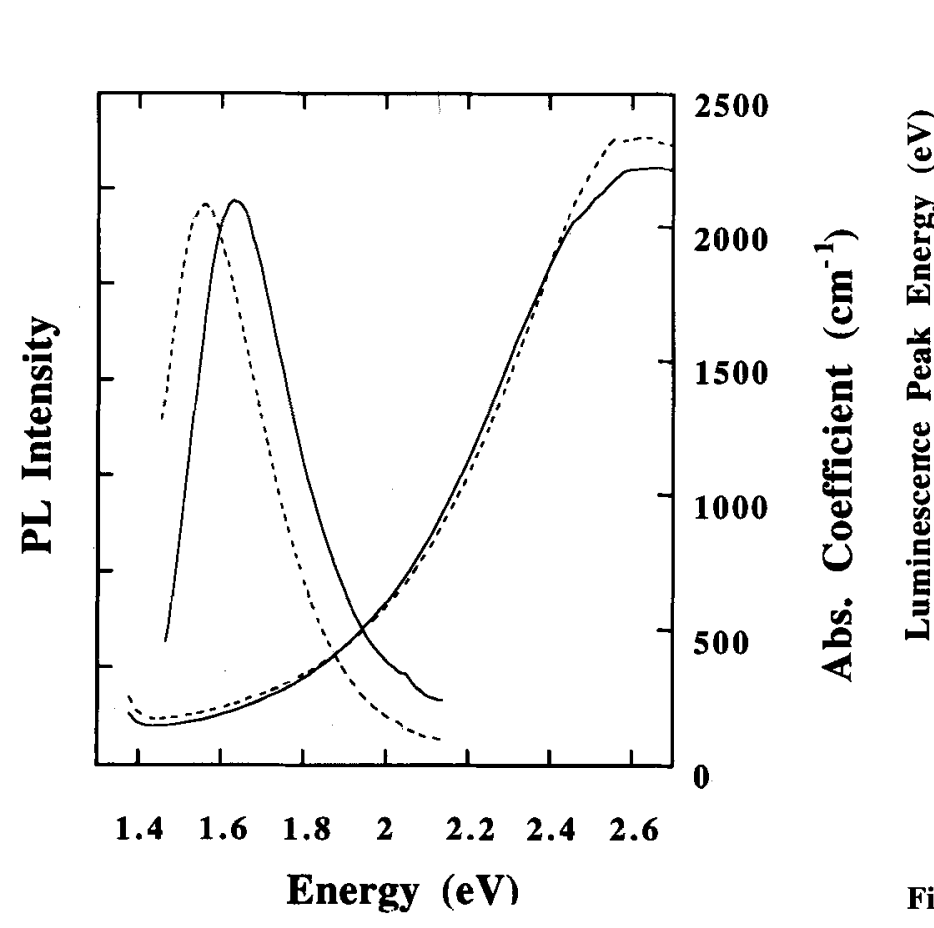

Figure 4. Comparison of optical absorption and photoluminescence spectra between H-PS (dotted line) and D-PS (solid line). luminescence (PL) spectra of H-PS and D-PS samples $\left(20 \mathrm{~mA} / \mathrm{cm}^{2}\right.$-samples) at room temperature. The PL spectra of these porous samples were measured in vacuum using $325 \mathrm{~nm}$ excitation light from a He-Cd laser. The calibration of the spectral sensitivity was performed using a standard halogen lamp. It was found that both H-PS and D-PS show the same absorption spectrum. This result shows another evidence that both H-PS and D-PS have the same size of nanostructure.

Despite the absorption spectra of both PS samples being the same, the peak energy of PL spectra were entirely different: $1.55 \mathrm{eV}$ for H-PS and $1.65 \mathrm{eV}$ for D-PS. To clarify the relation of PL to the size and the termination, we have studied the optical absorption and PL for samples with various band gaps, which were fabricated by changing the current densities and HF concentration (Lehmann et al 1993). With increasing dissolution current density and decreasing HF concentration, both H-PS and D-PS show higher energy shift of the absorption and PL spectra.

Figure 5 shows the relation between the bandgap energy $E_{\varepsilon}$ and PL peak energy $E_{\mathrm{p}}$ for H-PS and D-PS. The experimental plots of bandgap versus PL peak energies are shown by open squares for D-PS and solid circles for H-PS. At the same bandgap energy, the PL peak energy of D-PS is always higher than that of H-PS.

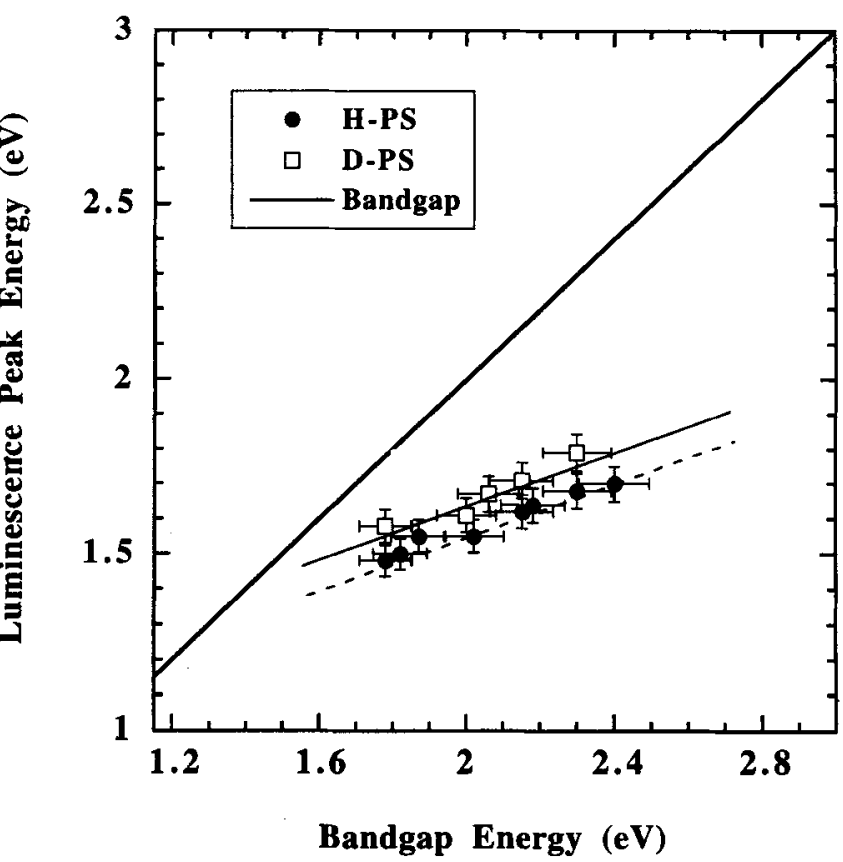

Figure 5. PL peak energy-plots as a function of the bandgap energies in H-PS (solid circles) and D-PS (open squares). The experimental plots are theoretically fitted with broken line for H-PS and solid line for D-PS. (The thick solid line shows the bandgap energy). 


\section{Analysis and discussion of optical properties}

A simple phonon assisted picture based on quantum confinement models cannot give a reasonable explanation for the above results. It is necessary to consider not only the bandgap upshift due to the quantum size effect but also the energy reduction due to the coupling of the confined carriers to the surface vibration of terminated atoms on nanocrystals.

Figure 6 shows a model in which the coupling between surface vibrations and the excited electrons leads to a strong localization of electrons accompanied by a structural relaxation of the surface. Here, $E_{g}$ is the bulk bandgap energy, $\Delta$ the total energy shift, and $\alpha$ and $\beta$ are the energy-shift ratios of the conduction band and the valence band, respectively. Based on our tight binding calculation, we find that the structural relaxation of $\mathrm{Si}-\mathrm{H}$ bonding causes relaxed excited states above $400 \mathrm{meV}$ from the conduction band of bulk Si crystal. As the size of the crystal becomes small, the bandgap will situate above this $\mathrm{Si}-\mathrm{H}$ relaxed states, and the excited electron gets trapped at this $\mathrm{Si}-\mathrm{H}$ or $\mathrm{Si}-\mathrm{D}$ bond surface. The PL process involves recombination of the trapped electron with the delocalized hole, and the upshift of PL peak energy versus size can be understood in terms of the upshift energy of the holes. The difference of the PL peak energy between $H$ and $D$ arises from the difference of the energies corresponding to $\mathrm{Si}-\mathrm{H}$ and $\mathrm{Si}-\mathrm{D}$ relaxations.

For a quantitative analysis, we consider the energy level shown in figure 6. The absorption energy and PL peak energies are described as

$$
\begin{aligned}
& E_{\mathrm{abs}}(L)=E_{\mathrm{g}}+(\alpha+\beta) \Delta(L), \\
& E_{\mathrm{pl}}^{\mathrm{H}}(L)=E_{\mathrm{g}}+\alpha \Delta(L)+\Delta^{\mathrm{H}}-\Delta_{\text {latt }}^{\mathrm{H}}, \\
& E_{\mathrm{pl}}^{\mathrm{D}}(L)=E_{\mathrm{g}}+\alpha \Delta(L)+\Delta^{\mathrm{D}}-\Delta_{\text {latt }}^{\mathrm{D}},
\end{aligned}
$$

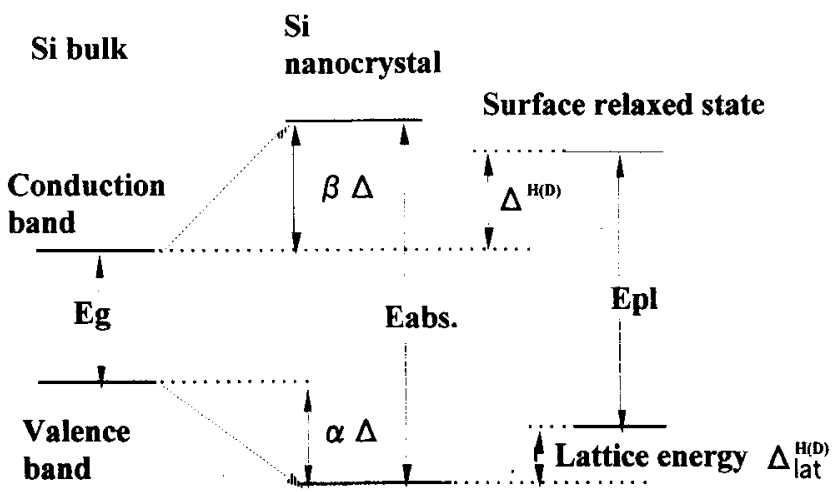

Figure 6. Schematic energy diagram of the radiative recombination process for H-PS and D-PS. where $\Delta(L)$ is the upshift energy by the quantum confinement effect, $\Delta^{\mathrm{H}}\left(\Delta^{\mathrm{D}}\right)$ the energy difference between the surface localized state and the bulk conduction band minimum, and $\Delta_{\text {latt }}^{\mathrm{H}}\left(\Delta_{\text {latt }}^{\mathrm{D}}\right)$ is lattice strain energy left behind during recombination process caused by the electron localization for H-PS (D-PS). From the observed dependence of the PL peak energy versus bandgap energy, we can determine the above parameters in (1), (2) and (3). We find $\alpha=0.38, \beta=0.62, \Delta^{\mathrm{H}}-\Delta_{\text {latt }}^{\mathrm{H}} \fallingdotseq 70 \mathrm{meV}$, $\Delta^{\mathrm{D}}-\Delta_{\text {latt }}^{\mathrm{D}} \fallingdotseq 160 \mathrm{meV}$. Thus, the difference of the PL peak energy can be calculated as $90 \mathrm{meV}$, and this energy difference is not affected by the size of the nanocrystal. The calculated results using these parameters are shown by a solid line for D-PS and a broken line for H-PS in figure 5, which well explain the PL peak energy difference between H-PS and D-PS.

\section{PL degradation}

It is generally known that H-PS exhibits strong degradation of not only PL but also EL, which is characteristic of hydrogen-terminated material (Collins and Tischler
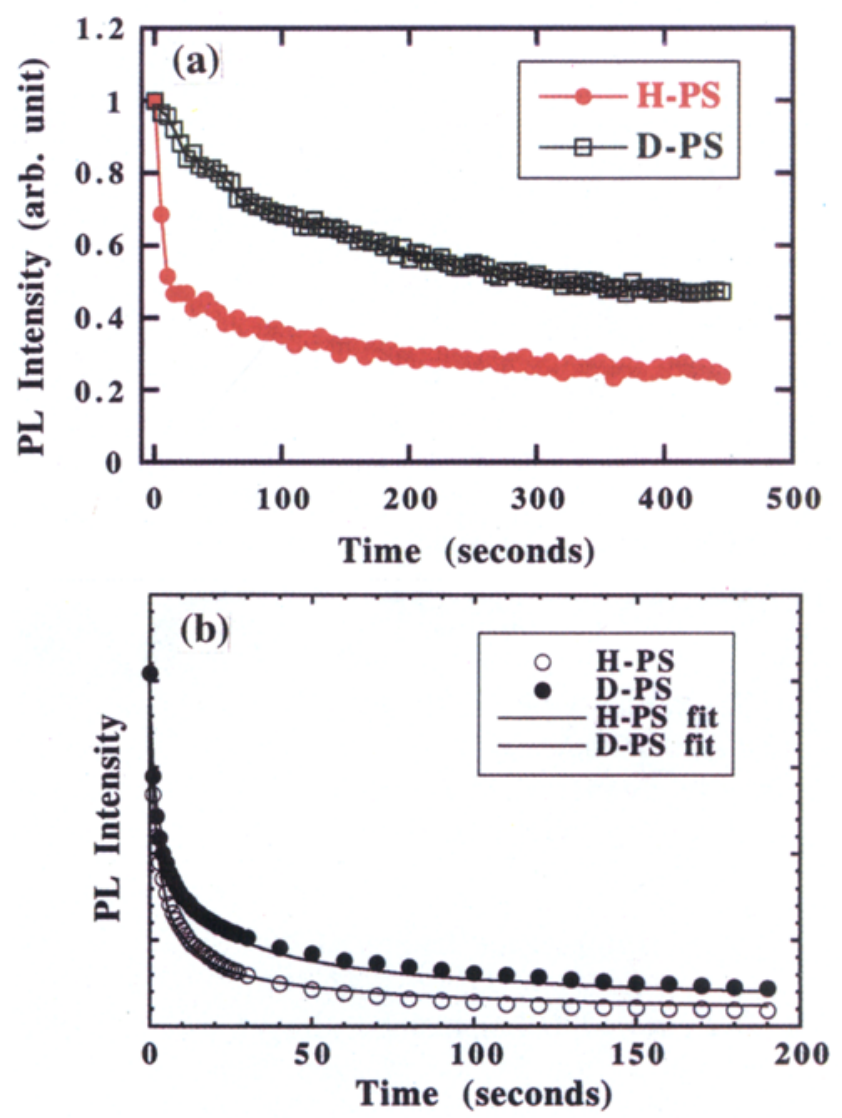

Figure 7. Comparative time-dependent degradation of PL in H-PS and D-PS fabricated from (a) p-type substrate and (b) n-type substrate. 
1993). In the above sections, we described both the nanostructures and the optical properties of H-PS and D-PS to clarify some fundamental aspects of light emitting porous $\mathrm{Si}$.

In this section, we will show that replacing hydrogen termination with deuterium greatly reduces the PL degradation by factors of $10-100$, and thus provides not only information regarding the luminescent mechanism but also a method of stabilization of EL devices.

PL degradation measurements were performed using a $\mathrm{He}-\mathrm{Cd}$ laser in oxidizing air ambient at room temperature. Figure 7 shows the comparative time-dependent degradation of PL intensity for H-PS and D-PS. It is found that the PL stability improves by a factor of 50 by replacing hydrogen with deuterium in porous $\mathrm{Si}$ fabricated from $p$-type substrates (figure $7 \mathrm{a}$ ). In porous $\mathrm{Si}$ fabricated from $n$-type substrates, the deuterium termination can also stabilize the PL intensity; however, they show significant PL quenching compared with that of $p$-type substrates as shown in figure $7 \mathrm{~b}$.

Figure 8 shows the ratio of FTIR spectra (performed for H-PS and D-PS) between as-prepared and 5 min light exposed samples. It is found that the absorption related with oxygen termination increases, for example, the $\mathrm{Si}-\mathrm{O}-\mathrm{Si}$ asymmetric stretching mode around $1050 \mathrm{~cm}^{-1}$ as shown in figure 8a, whereas the absorption related to hydrogen termination does not change by light exposure (figure 8b). Furthermore, we found that the deuterium termination can significantly reduce the rate of lightinduced oxidation compared with hydrogen termination as shown in figure $8 \mathrm{c}$. These results suggest that the light soaking accelerates the increase of $\mathrm{Si}-\mathrm{O}-\mathrm{Si}$ absorbance without hydrogen desorption and that the oxidation rate of D-PS is smaller than that of H-PS under the same photoinduced oxidation conditions. In $\mathrm{N}_{2}$ atmosphere or in vacuum, PL does not show degradation, and this is correlated with the IR absorption spectrum showing no difference between as-prepared and 20 min light soaking. Therefore, we can conclude that the PL quenching occurs by light-induced oxidation and that the oxidation does not occur by substitution of the surface hydrogen but by insertion into the back bond of the surface Si-H bond. The reason why the IR absorption-change of D-PS is smaller than that of H-PS is not clearly understood. One possible explanation is the difference in the vibrational frequency affects the oxidation rate.

\section{Electroluminescence from D-PS}

In $\S 4$, we showed that replacing hydrogen with deuterium greatly reduces the PL degradation. Especially, better PL-stabilization was obtained from D-PS fabricated from $p$-type substrates. However, it is generally known that PS prepared from highly doped $n$-type substrates show intense EL. Thus, here, we will describe the electrical properties of LEDs based on D-PS anodized from highly doped $n$-type substrate.

Figure 9 shows the current-voltage $(J-V)$ characteristics of both the ITO/D-PS and ITO/H-PS junctions. Open circles and solid circles correspond to the $J-V$ charac-

(a)

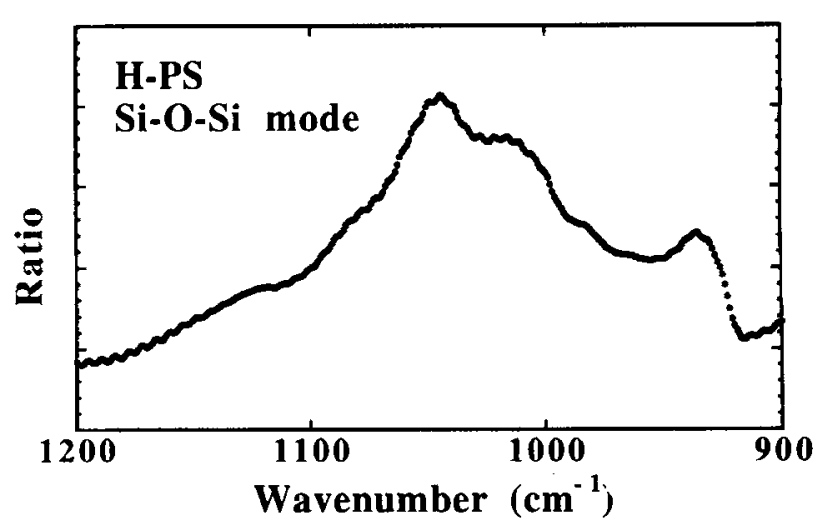

(b)

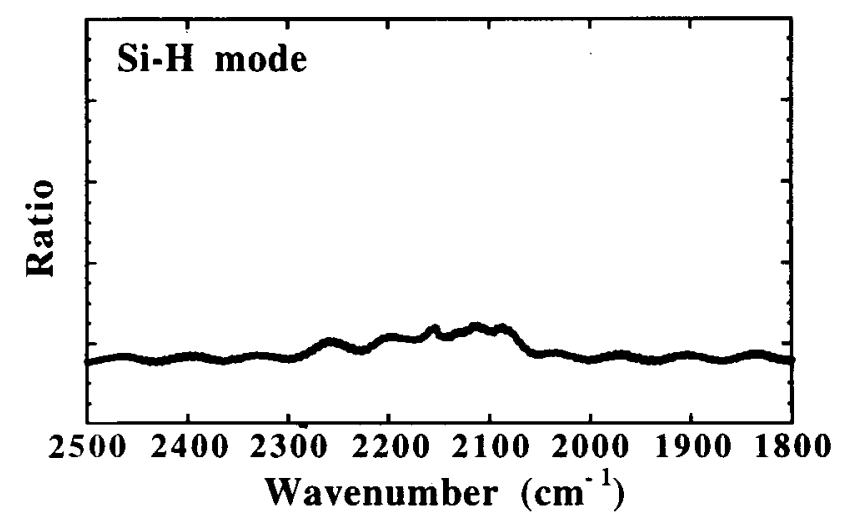

(c)

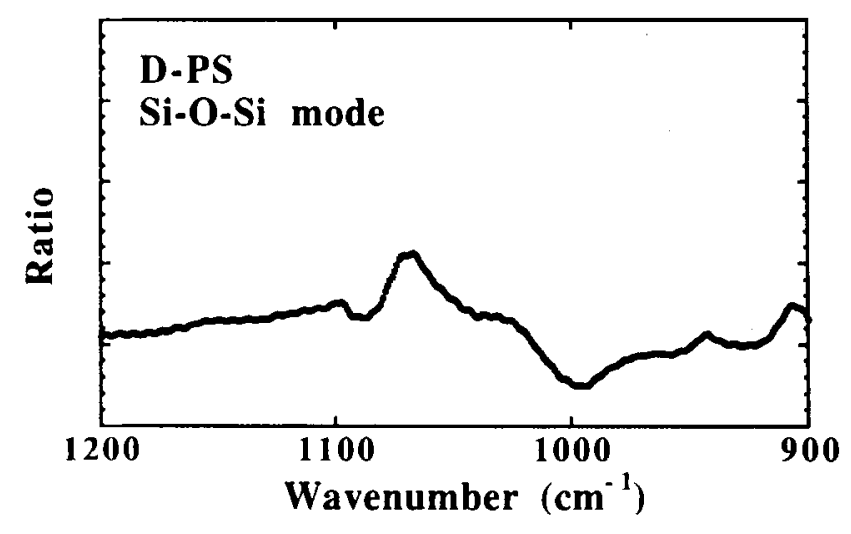

Figure 8. Ratio of FTIR spectra between as prepared and 5 min light soaked porous $\mathrm{Si}$. (a) Si-O-Si stretching mode of $\mathrm{H}-\mathrm{PS}$, (b) Si-H stretching mode, and (c) $\mathrm{Si}-\mathrm{O}-\mathrm{Si}$ stretching mode of D-PS. 
teristics of H-PS and D-PS, respectively. Clear rectifying junction behaviours were observed for both samples. However, the rectifying ratio is relatively low, being of the order of 10 for ITO/H-PS and 25 for ITO/D-PS at the applied bias voltage $\left(V_{\mathrm{b}}\right)$ of $10 \mathrm{~V}$. The calculated ideality factors $(n)$ of these diodes can be determined by the standard diode equation, and at biases $>3 \mathrm{~V}$, the data corresponds to an ideality factor $n$ of 22 for ITO/H-PS and 50 for ITO/D-PS. However, at lower applied bias voltages $\angle 3 \mathrm{~V}$, another branch is observed with a smaller ideality factor $n=10$ for H-PS and $n=28$ for D-PS, those are rather high values. The relatively low rectifying ratio and the high ideality factors may originate from the high resistivity of the thick porous layer (thickness $40 \mu \mathrm{m}$ ) and/or the charging of the interface states at the oxide/PS surface.

Figure 10 shows plots of the EL intensity as a function of the diode current for ITO/D-PS- (solid circles) and ITO/H-PS- (open circles) junctions, respectively. Both junctions show light emission when the forward bias voltage becomes about $5 \mathrm{~V}$. It is clear that ITO/D-PS junctions show higher EL efficiency than ITO/H-PS junctions. The reason for this has not been clarified. However, we consider that the carrier-injection efficiency is correlated with the vibrational frequency of surface terminated atoms-the lower vibrational frequency of $\mathrm{Si}-\mathrm{D}$ compared to $\mathrm{Si}-\mathrm{H}$ reduces the number of scattered

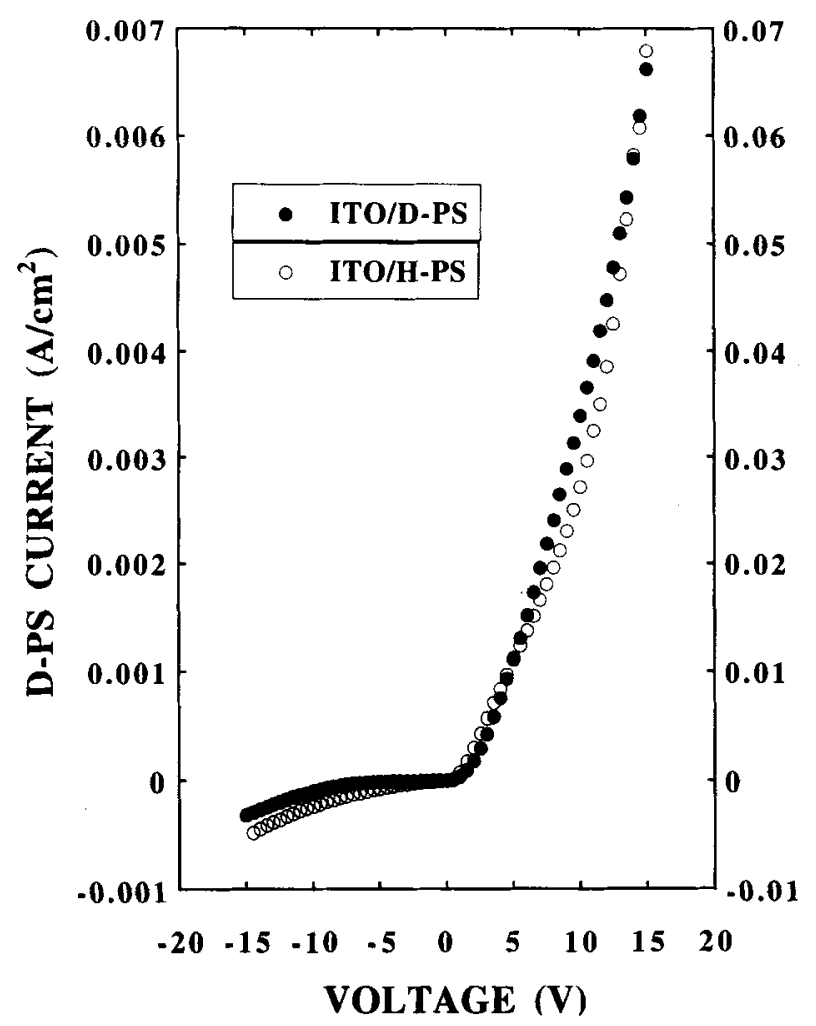

Figure 9. Current-voltage characteristics of the ITO/D-PS (solid circles) and ITO/H-PS (open squares) junctions. carriers being injected through the electrode into the luminescent porous layer.

As the current increases, the dependence of the EL intensity $(I)$ on the current, given by $I \propto J^{\mathrm{n}}$, changes its power $(m)$ from 5 to 3 in D-PS, and from 4 to 1 in H-PS. The superlinear dependence of EL intensity at lower current levels indicates that the recombination is dominated by space-charge-limited-currents. When the applied positive bias voltage exceeds $10 \mathrm{~V}$, a uniform visible EL is observed from both D-PS and H-PS diodes through the ITO electrode. Both the PL and EL spectral peaks of a D-PS layer situate at the higher energy side compared with those of H-PS layer. This result is the same as was discussed in $\S 3$, and it can be explained from the difference of the surface termination (especially vibrational frequency) between $\mathrm{Si}-\mathrm{H}$ and $\mathrm{Si}-\mathrm{D}$. The ITO/D-PS device had a relatively high quantum efficiency of the order of $0.1 \%$. The similarity between the PL and EL spectra indicates that the EL emission is due to radiative recombination of electrons and holes generated in the active PS layer.

Figure 11 shows the time-dependent degradation of the EL-intensity for H-PS and D-PS. The EL degradation

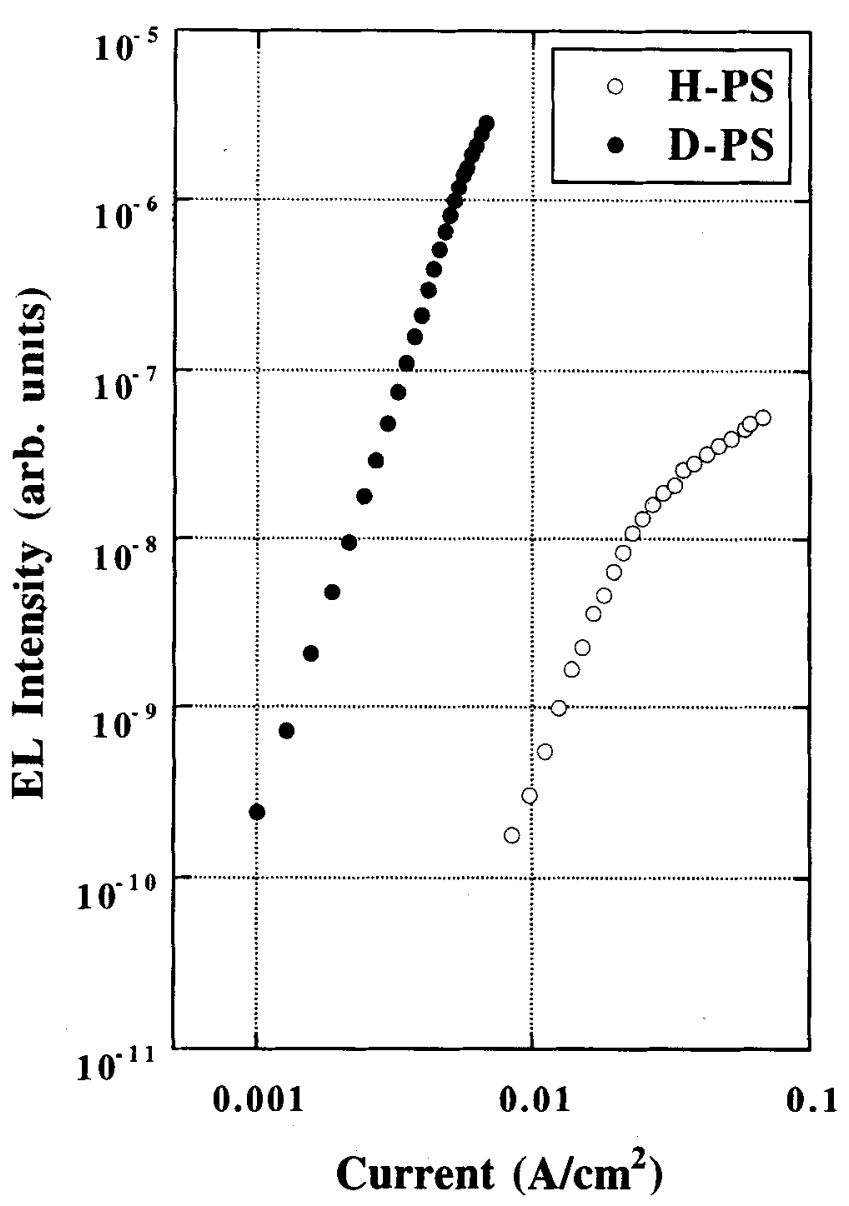

Figure 10. Dependence of EL intensity on device current for ITO/D-PS (solid circles) and ITO/H-PS (open squares) junctions. 


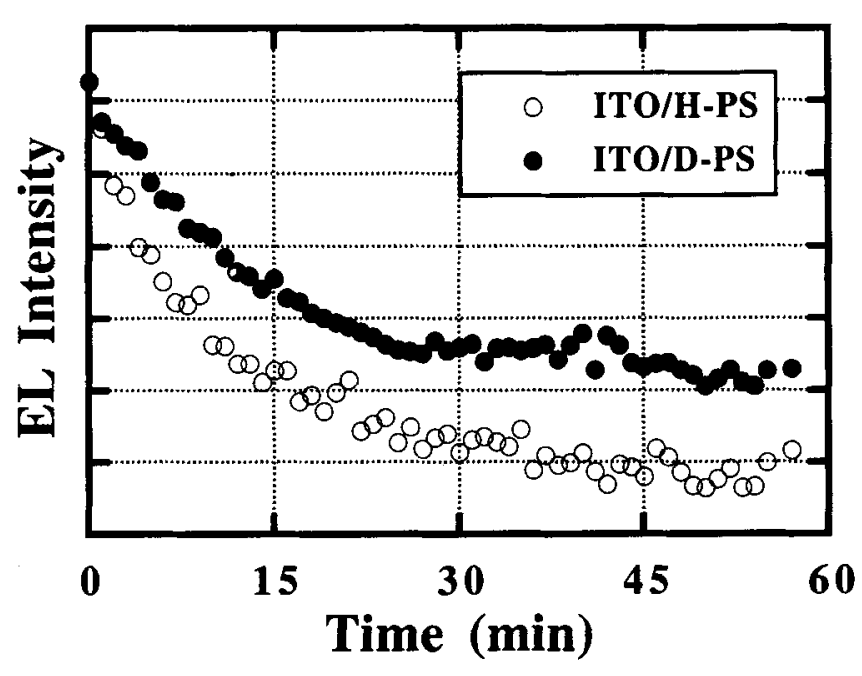

Figure 11. Time dependent degradation of EL intensity for H-PS (open circles) and for D-PS (solid circles).

measurements were performed under the conditions of $\mathrm{CW}$ operation and oxidizing air ambient at room temperature. It is found that the EL intensity is more stable than the PL intensity and that the replacement of hydrogen with deuterium also reduces the EL-degradation. For EL degradation, the capping layer of the ITO-electrode can prevent oxidation of the PS surface, thus the EL degradation was reduced compared to the PL degradation.

\section{Conclusion}

In $\S \S 2$ and 3 , we have described the surface termination dependence of the PL peak energy for porous Si. The experimental results strongly suggest that, in addition to the bandgap upshift due to the quantum size effect, there is a significant energy reduction due to the coupling of the confined carriers to the surface vibration of terminated atoms on nanocrystals. We have argued the difference of the PL peak energy from the point of view of trapping of the conduction electrons at a surface $\mathrm{Si}-\mathrm{H}$ or $\mathrm{Si}-\mathrm{D}$ bond. Our model clarified that the PL process involves recombination of trapped electron with the delocalized hole.

In $\S \S 4$ and 5, we have shown that the deuterium termination gives not only the reduction of PL and EL degradations but also efficient EL to porous Si. Therefore, replacement of hydrogen with deuterium is very promising for the fabrication of optoelectronic devices from the point of view of the compatibility of carrier injection and device stability, which is not possible by oxygen termination. Key problems, such as stability over several days and output power, are to be solved.

\section{References}

Campbell I H and Fauchet P M 1984 Solid State Commun. 58 739

Canham L T 1990 Appl. Phys. Lett. 571046

Chabal Y J and Raghavachari K 1984 Phys. Rev. Lett. 53282

Collins R T and Tischler M A 1993 IEEE Circuits and Devices 922

Gupta P, Colvin V L and George S M 1988 Phys. Rev. B37 8234

Hardeman R W, Beale M I J, Gasson D B, Keen J M, Pickering C and Robbins D J 1985 Surf. Sci. 152/153 1051

Lehmann V, Jobst B, Muschik T, Kux A and Petrova-Koch V 1993 Jpn J. Appl. Phys. 322095

Matsumoto T, Masumoto Y, Nakagawa T, Hashimoto M, Ueno K and Koshida N 1997a Jpn J. Appl. Phys. 36 L1089

Matsumoto T, Masumoto Y, Nakashima S and Koshida N $1997 \mathrm{~b}$ Thin Solid Films 29731

Pankov J I and Johnson N M 1991 Hydrogen in semiconductor (eds) J I Pankov and N M Johnson (New York: Academic Press)

Richter H, Wang Z P and Ley L 1981 Solid State Commun. 39625 